\title{
Elias Artista oder Das Überflüssigwerden des Wissens in Kuhlmanns Kühlpsalter
}

\section{Elias Artista}

Im 16. und 17. Jahrhundert finden sich in verschiedenen theologischen Diskursen endzeitliche Gedankenfiguren, die auf Joachim von Fiore (1130/1135-1202) zurückgehen und nun eine deutliche Renaissance erfahren. ${ }^{1}$ Die christliche Heilsgeschichte ist bei Joachim gemäß der Trinität dreigeteilt: Vater, Sohn, Heiliger Geist. Der Übergang von der Zeit des Vaters auf den Sohn ist mit dem Übergang vom Alten zum Neuen Testament erfolgt. Jetzt aber glaubt man sich am Übergang vom "secundo« statu (dem des Sohnes) zum »tertio statu " (dem des Heiligen Geistes) ${ }^{2}$ - und zwar als Realisierung von endzeitlichen Potenzialen, die schon in den vorhergegangenen, jeweils konkordanten, Status angelegt waren. Zentralfigur für diesen zweiten Übergang ist der Prophet »Elia«, der nach Joachims Vorstellungen bei seiner Wiederkunft die dritte Zeit ankündigt. ${ }^{3}$

Warum bringen Joachim von Fiore und die frühneuzeitlichen Autoren, die sich seiner Gedankenfiguren bedienen, den Propheten Elia argumentativ derart in Stellung? Elia agiert, biblisch gesprochen, auf mehreren Zeitebenen:

1 Dieser erste, grundlegende Teil des Aufsatzes fasst die Ergebnisse eines Kapitels meiner Habilitationsschrift, Vf., Nachfolge Christi - Nachahmung der Natur. Himmlische und natürliche Magie bei Paracelsus, im Paracelsismus und in der Barockliteratur (Scheffler, Zesen, Grimmelshausen), Hamburg 2007, S. 38-48, zusammen. Einige Passagen sind textidentisch. Vgl. hierzu auch Joachim Telle/Wilhelm Kühlmann (Hg.), Corpus Paracelsisticum, Tübingen 2001ff., Bd. I, S. 168f. (im Folgenden unter der Sigle CP zitiert); Richard Bauckham, Art. Chiliasmus IV, in: Gerhard Müller u.a. (Hg.), Theologische Realenzyklopädie, Berlin 1977-2010, Bd. VII, S. 737-745, hier S. 737f., sowie Wilhelm Schmidt-Biggemann, Apokalypse und Philologie, Wissensgeschichten und Weltentwürfe der Frühen Neuzeit, hg. von Anja Hallacker/Boris Bayer, Göttingen 2007, S. 201-203.

2 Joachim von Fiore, Liber de concordia noui ac veteris testamenti, hg. von E. Randolph Daniel, Philadelphia 1983, S. 158 (21rb). Hierzu nach wie vor grundlegend, Alois Dempf, Sacrum Imperium, Geschichts- und Staatsphilosophie des Mittelalters und der politischen Renaissance, Darmstadt ${ }^{2}$ 1954, S. 272-279; Herbert Grundmann, Studien über Joachim von Fiore, Darmstadt 1966, S. 58f.

3 Joachim von Fiore, Liber de concordia, S. 161 (22ra). Vgl. hierzu Bernard McGinn, Joachim of Fiore's Tertius status, S. 219-222, in: Ders., The Calabrian Abbot, Joachim of Fiore in the History of Western Thought, New York 1985, S. 161-203. 
Er ist einerseits laut Altem Testament ein Prophet, der gegen den Baalskult kämpft. Insofern ist er dem ersten Zeitalter zuzurechnen. Gleichzeitig wird er gemäß Mal 3,23f. am großen und schrecklichen Tag des Herrn noch einmal wiederkommen und als Gerichtsbote die letzte Gnadenzeit Gottes ankündigen. Diese Vorstellung wird im Neuen Testament aufgenommen; z.B. in Mt 11,14; 16,14; 17,10-13; Joh 1,21. In diesem Falle ist es Johannes, der als Figura Eliae ('Figurar im Sinne Auerbachs verstanden) ${ }^{4}$ fungiert und damit Jesus Christus ankündigt.

Bemerkenswert ist nun, dass dieser, das dritte Zeitalter ankündigende, Elia im Paracelsismus beinahe ausschließlich mit einem Beinamen genannt wird. Die Anhänger des Paracelsus apostrophieren den Propheten nämlich als "Eliam artistam«. ${ }^{5}$ Damit wird eine bemerkenswerte Variante im endzeitlichen Denken der Frühen Neuzeit augenscheinlich gemacht: Ein 'Artistar ist in der Frühen Neuzeit, im Gegensatz zum theoretisch oder grundlagenorientiert arbeitenden Scientisten, ein praktischer, technischer oder anwendungsbezogener Wissenschaftler. ${ }^{6}$ Elias Artista ist demzufolge nicht nur der Prophet für die tausendjährige Gottesherrschaft auf Erden, sondern der Prophet, der das Erreichen des Endes der Zeit durch praktische Wissenschaften ankündigt. Er steht also nicht nur für Offenbarung schlechthin, sondern für eine Offenbarung allen technischen Wissens.

Die Gedankenfigur des Elias Artista ist bei fast allen namhaften Paracelsisten zu finden: Oswald Croll spricht beispielsweise davon, dass "Elia der Artist" ("Helias Artista") als "Reparator omnium" im "dritten seculo«, d.h. dem des Heiligen Geistes, erscheinen wird. Jacob Böhme, um ein weiteres

4 Vgl. zur Figura-Lehre allgemein Erich Auerbach, Figura, in: Ders., Neue Dantestudien, Sacrae scripturae sermo humilis, Figura, Franz von Assisi in der Komödie, Istanbul 1944, S. 11-71, und zur Figura-Lehre im Barock, am Beispiel von Harsdörffer, Vf., Warum Frauen mit dem Teufel schlafen, Männer hingegen mit ihm Verträge abschließen wollen. Diabolische Figurenlehre in Harsdörffers 'Schau-Plätzen`, in: Joel B. Lande/ Robert Suter (Hg.), Dynamische Figuren. Gestalten der Zeit im Barock, Freiburg i.Br. 2013, S. 77-100.

5 Michael Toxites, Widmungsvorrede an Pfalzgraf Ludwig, 15.1.1574, zu: Bernardus Trevisanus: Von der hermetischenn Philosophia, in: CP II, 281 [Hervorh. MB].

6 Vgl. hierzu Barbara Mahlmann-Bauer, Einleitung, in: Dies. (Hg.), Artes et scientiae, Künste und Wissenschaften - im Verhältnis zur Natur, Wiesbaden 2004, Bd. I, S. 11-64.

7 Oswald Croll, Basilica chymica, oder Alchymistisch königlich Kleynod [...], ohne Angabe des Übersetzers, Frankfurt a.M. 1623, S. 5; Ders., Basilica chymica, Continens philosophicam propriâ laborum experientiâ confirmatam descriptionem et usum remediorum chymicorum [...], Hildesheim/Zürich/New York 1993, S. 7. 
Beispiel zu nennen, streut in seine Texte die Formulierung "wie es der Artista sehen wird ${ }^{8}$ ein (ein Ereignis, das in seinen Augen unmittelbar bevorsteht). Die Gedankenfigur des Elias Artista ist jedoch keine Erfindung des Paracelsismus, sondern findet sich bereits bei dem Autor, auf den sich der Diskurs bezieht. Angeregt durch seine Teilnahme an der Prognostiken-Flut der 20er Jahre ${ }^{9}$ hatte bereits Paracelsus selbst epistemo-apokalyptische Hoffnungen gehegt ${ }^{10}$ und diese seinerseits unter dem Begriff des Elias Artista gefeiert, z.B. wenn er in Von den natïrlichen Dingen die "zeit der künst HElia« ankündigt, in der "got offenbar werden« lässt, was jetzt noch »verborgen« ist. ${ }^{11}$

Der Bezug des Paracelsismus auf Paracelsus in der Frage des Elias Artista ist ein zentraler Eckpfeiler des Diskurses, denn wenn von Offenbarung allen Wissens die Rede ist, dann ist im Paracelsismus der Wissensprozess gemeint, den Paracelsus durch seine alchemotheologische Arbeit angestoßen hat und den die Paracelsisten weiter fortführen; ein Wissensprozess, der es erlaubt, auch in epistemo-apokalyptischer Hinsicht eine, wie die Paracelsisten glauben, begründete Form der endzeitlichen Naherwartung an den Tag zu legen. Paracelsus hat, als Erster, wie sich das für einen Diskursgründer gehört, das wahre Wissen vom Verborgenen an die Oberfläche geholt und dadurch einen Prozess angestoßen, der durch seine Schüler fortgesetzt wird - und zwar immer schneller und schneller, bis dieser Prozess im dritten Status abgeschlossen sein wird.

Genau das führt Michael Toxites, ein weiterer Paracelsist, aus, wenn er Elias Artista als Ankündiger und Eröffner der "zeit [...] / da es alles offenbar werden soll«, bezeichnet. Offenbar werden soll das Wissen, das sich bisher schon weitgehend, aber eben nicht vollständig offenbar gezeigt hat, nämlich das paracelsische. Genauer gesagt ist mit dem Auftritt von Elias Artista auch eine Neuinterpretation des in den paracelsischen und paracelsistischen Texten gespeicherten Wissens der Natur verbunden. Bis jetzt, so Toxites' Vorstellungen weiter, müssen die Menschen noch in "Bücher[n] [...] vben / vnnd

8 Jakob Böhme, De signatura rerum, in: Ders., Werke, hg. von Ferdinand van Ingen, Frankfurt a.M. 1997, S. 676f. (im Folgenden: W).

9 Vgl. hierzu Charles Webster, Paracelsus. Medicine as Popular Protest, in: Ole Peter Grell/ Andrew Cunningham (Hg.), Medicine and the Reformation, London/New York 1993, S. 57-77, hier S. 59f.

10 Hierzu Walter Pagel, The Paracelsian Elias Artista, in: Rosemarie Dilg-Frank (Hg.), Kreatur und Kosmos. Internationale Beiträge zur Paracelsus-Forschung, Stuttgart/New York 1981, S. 6-19, hier S. 12.

11 Paracelsus, Sämtliche Werke, Bd. I/2, hg. von Karl Sudhoff, München 1929ff., S. 163 (im Folgenden SW). Vgl. hierzu auch Carlos Gilly, Johann Arndt und die sdritte Reformation im Zeichen des Paracelsus, in: Nova Acta Paracelsica NF 11 (1997), S. 60-77, hier S. 62f. 
suchen«. Wenn Elias Artista kommt, wird er jedoch »erklären «, ${ }^{12}$ was, wie parallel bei einem zeitgenössischen Paracelsisten, Alexander von Suchten, zu lesen ist, durch die Bücher noch »verborgen« ist, indem er »dieselbigen außlegt«. 13

Die hiermit formulierte Gedankenfigur basiert auf einer intellektuellen Überblendung der Vorstellung von der Offenbarung als Ende der Zeit mit der alchemischen Theorie, dass alles, was verborgen ist, offenbar werden muss: ${ }^{14}$ Das Buch der Natur, Paracelsus' Bücher über dieses Buch, die Bücher über Paracelsus' Bücher durch die Paracelsisten; diese Abfolge ist für Letztere ein integraler Bestandteil des Prozesses der Offenbarmachung von bisher verborgenem Wissen. Vor dem skizzierten chiliastischen Horizont gehört das wissenschaftliche Arbeiten in und mit Büchern jedoch noch in das zweite Zeitalter, da hier der Prozess des Offenbarwerdens noch im Gange ist. Im dritten Zeitalter wird hingegen alles Wissen offenbar sein. Und das bedeutet wiederum, dass der Prozess der Wissensvermittlung und -auslegung in und durch Bücher überflüssig oder eben unnütz werden wird. Und genau das, also das Ende des buchgestützten Wissens, zeigt der TechnikProphet Elias Artista an.

Für Paracelsus und die Paracelsisten, gerade in ihrer Eigenschaft als Autoren oder Editoren von Büchern, ist diese Gedankenfigur weitgehend unproblematisch, da Paracelsus zwar mit Elias Artista in Verbindung steht, aber, von wenigen Ausnahmen abgesehen, ${ }^{15}$ nicht mit ihm identifiziert wird. Gott habe durch »Paracelsum», das "Liecht" der Medizin erstlich angezündet, schreibt beispielsweise Michael Toxites. Aber das heißt eben noch nicht, dass es jetzt schon ganz hell wäre. Gott will uns jetzt, wie Toxites weiter

12 Michael Toxites: Widmungsvorrede an Pfalzgraf Ludwig, 15.1.1574, zu: Bernardus Trevisanus: Von der hermetischenn Philosophia, in: CP II, S. 281.

13 Alexander von Suchten, Chymische Schriften Alle, Frankfurt a.M. 1680, S. 244. Vgl. hierzu Carlos Gilly, Johann Arndt, S. 63.

14 »dan nichts ist, das verborgen bleibe und nit geoffenbaret, es muß alles herfür, geschöpf, natur, geist, bös und guts, außen und innen, und all künst und alle doctrinen, ler und was beschaffen ist«, heißt es beispielsweise bei Paracelsus, Philosophia magna, SW I.14, 131, und zwar, wie es weiter heißt, »ehe das der jüngst tag kompt« (ebd., S. 150f.). Dieser alchemische Leitsatz bezieht sich auf Lk 8,17: "Non enim est occultum quod non manifestetur» (Zit. nach Biblia sacra, iuxta Vulgatam versionem, hg. von Robert Weber/Roger Gryson, Stuttgart ${ }^{52007 . ~[H e r v o r h . ~ M B]) . ~}$

15 Vgl. hierzu Adam von Bodenstein, Widmungsvorrede an Erzherzog Ferdinand II., 24.12.1571, zu: Paracelsus, Metamorphosis, in: CP I, 460, der sein Schreiben mit einem Hinweis auf die "lucubrationes", also die Nachtarbeiten, "Aureoli Paracelsi / Helie artiste« beginnt [Hervorh. MB]. 
schreibt, »länger nit im finstern [...] lassen«. Jetzt soll alles andere »offenbar werden«. ${ }^{16}$

Die Betonung liegt bei Toxites eindeutig auf dem Werden. Denn es ist leicht nachzuvollziehen, dass die Selbstrechtfertigung des Paracelsismus auf der Prämisse beruht, dass mit Paracelsus die Offenbarungsarbeit allen Wissens zwar angefangen hat, keineswegs jedoch abgeschlossen wurde. Genau hier liegt der Selbstanspruch der Paracelsisten, nämlich das Werk des Paracelsus weiter voranzutreiben. Dementsprechend behält Elias Artista im Paracelsismus meist eine futurische Dimension. So formuliert es ja auch Böhme: „Wie es der Artista sehen wirdk (s.o.).

In dem Augenblick aber, in dem sich ein Autor - um zum eigentlichen Gegenstand meines Aufsatzes, nämlich zu dem Barockpoeten Quirinus Kuhlmann (1651-1689), zu kommen - selbst für Elias Artista oder für einen wie auch immer gearteten Eröffner des neuen Zeitalters hält, entsteht ein Problem des paradoxalen Selbstbezugs. Wenn der Artista das Überflüssigwerden allen bisherigen buchgestützten Wissens in seiner traditionellen, prozessualen Form (Sichtbarmachen des Unsichtbaren) ankündigt bzw. eröffnet, ein Autor wie Kuhlmann jedoch selbst dieser Artista zu sein behauptet und das Zeitalter für angebrochen hält, dann widerspricht das Buch, das er gerade schreibt, performativ seinem Inhalt, denn im joachimitischen dritten Zeitalter ist alles buchgestützte Wissen unnütz, weil alles Wissen offenbar geworden ist und nicht mehr aus der Dunkelheit zwischen den Buchdeckeln gezogen werden muss.

\section{Kuhlmann und der Artista}

Wer ist Quirinus Kuhlmann? Es handelt sich um einen aus Breslau stammenden, in seiner (Jenaer) Anfangszeit literarisch ambitionierten Autor, dabei in seiner Lyrikproduktion durchaus dem barocken Bildungsideal schlesischer Prägung (mit Nürnberger Anleihen, wie man hinzufügen muss) verpflichtet. ${ }^{17}$ Kuhlmann hatte anscheinend im Jahre 1673/1674 in Leiden

16 Michael Toxites, Widmungsvorrede an Graf Philipp von Hanau-Lichtenberg, 25.7.1565, zu: Paracelsus, Drey Bücher, CP II, 72 [Hervorh. MB]. Zur Tradition dieser Sentenz (z.B. bei Arnald von Villanova), vgl. Telle/Kühlmann, Kommentar, CP II, S. 86.

$17 \mathrm{Zu}$ Kuhlmanns Breslauer und Jenaer Zeit, vgl. Walter Dietze, Quirinus Kuhlmann, Ketzer und Poet. Versuch einer monographischen Darstellung von Leben und Werk, Berlin 1963, S. 25-67; zu seiner schlesischen Prägung, ebd., S. 74; zu seinen Kontakten nach 
- und zwar unter dem Einfluss der Lektüre verschiedener Schriften Jakob Böhmes - eine Art intellektuelles Erweckungserlebnis, das sich vorderhand im Neubegeisterten Böhme (ED 1674), ${ }^{18}$ dann aber in seinem Hauptwerk, dem Kühlpsalter, niederschlägt (ED 1684-1686, mit Texten seit den 70er Jahren) ${ }^{19}$ In diesem letzteren Text werden, auf Basis eines in gebundener Rede gehaltenen Reisetagebuchs, wie man es probehalber nennen möchte, ${ }^{20}$ aus dem Luthertum stammende, aber über dieses entschieden hinausgehende, ja sich gegen es wendende, ${ }^{21}$ chiliastische ${ }^{22}$ Visionen entwickelt. Zentraler Stichwortgeber ist nach wie vor Böhme, über den Kuhlmann Zugang zum paracelsisch-paracelsistischen Wissen findet.

Nicht alle mystischen Gedichte sind von Kuhlmann. In einigen Fällen handelt es sich um Übersetzungen von Texten des Juan de la Cruz aus dem Spanischen bzw. Lateinischen. ${ }^{23}$ Doch beinahe immer ist es Kuhlmann selbst, der im Zentrum der Ausführungen steht bzw. sitzt. Er ist der letzte Prophet und, in dem eben ausgeführten joachimischen Sinne, Eröffner eines neuen Zeitalters, also einer Gottesherrschaft auf Erden. Er präsidiert über verschiedene Kühlpropheten, also Autoren, die das, was er zu sagen und zu eröffnen hat, vorweggenommen bzw. angefangen haben. Bei Letzteren handelt es sich vor allem um den mährischen Prediger und Visionär Nikolaus/Mikulás

Nürnberg, ebd., S. 79-82. Siehe dazu auch Claus Viktor Bock, Quirinius Kuhlmann als Dichter. Ein Beitrag zur Charakteristik des Ekstatikers, Bern 1957, S. 7-15.

18 Hierzu Walter Dietze, Kuhlmann, S. 100-102; S. 109-113; Wilhelm Schmidt-Biggemann, Apokalypse, S. 190f. Vgl. zum Verhältnis Böhme/Kuhlmann auch Ferdinand van Ingen, Jacob Böhme und die schlesischen Dichter Daniel von Czepko, Johannes Scheffler und Quirinus Kuhlmann, in: Hartmut Laufhütte (Hg.), Heterodoxie in der Frühen Neuzeit, Tübingen 2006, S. 243-267, hier S. 262-265.

19 Vgl. Walter Dietze, Kuhlmann, S. 263; Wilhelm Schmidt-Biggemann, Apokalypse, S. 206.

20 Hierzu auch Harald Haferland, Heilsbedeutung und spekulative Alchemie. Böhme-Rezeption bei Quirinus Kuhlmann, in: Wilhelm Kühlmann ( $\mathrm{Hg}$.), Offenbarung und Episteme. Zur europäischen Wirkung Jakob Böhmes im 17. und 18. Jahrhundert, Berlin 2012, S. 143-164, hier S. $145 f$.

21 Hierzu Walter Dietze, Kuhlmann, S. 129.

22 Zum chiliastischen, also nicht i.e.S. apokalyptischen, Denken Kuhlmanns, vgl. Walter Dietze, Kuhlmann, S. 128; S. 135-137; S. 177-184. Vgl. zum Chiliasmus in der Frühen Neuzeit allgemein, seinen Rekurs auf Offb 20,1-15 und seine spezifischen Interpretation dieser Bibelstelle (ewigwährendes Gottesreich statt Zwischenstation), Otto Böcher, Art. Chiliasmus I, in: Theologische Realenzyklopädie, hg. von Gerhard Müller u.a., Berlin 1977-2010, Bd. VII, S. 723-729, und Richard Bauckham, Art. Chiliasmus IV, S. 738.

23 Hierzu Leonard Forster, A.A. Parker, Quirinus Kuhlmann and the Poetry of St. Johan of the Cross, in: Bulletin of Hispanic Studies 25 (1958), S. 1-23; Sibylle Rusterholz, Klarlichtige Dunkelheiten. Quirinus Kuhlmanns 62. Kühlpsalm, in: Martin Bircher (Hg.), Deutsche Barocklyrik, Gedichtinterpretationen von Spee bis Haller, Bern 1973, S. 225-265, hier S. 233-253. 
Drabitz (1587/88-1671; auch Drabik, lt. Drabicius), die polnische Adlige Christina/Krystyna Poniatovska (1610-1644; auch Ponicowskin, lt. Poniatovia) sowie Christoph/Kryštofer Kotter (1585-1647; auch Kötter, lt. Kotterus), einen Gerber aus Sprottau, die bzw. deren Visionen Kuhlmann aus Johann Amos Comenius' Veröffentlichung Lux in bzw. E tenebris (1657 bzw. 1665/67) kennt. Es handelt sich um Prophetien aus der Zeit des Dreißigjährigen Kriegs, welche einen protestantischen Endsieg über den Katholizismus und den Untergang des Hauses Habsburg voraussagen. ${ }^{24}$ Dass beides nicht eingetreten ist, scheint der Attraktionskraft der Texte keinen Abbruch getan zu haben.

Wie Kuhlmann die drei Genannten einordnet, wird aus der Vorrede zum Vierten Buch des Kühlpsalters deutlich: Über Drabitz schreibt er beispielsweise: "Alle seine Worte werden erfüllet, und er den Propheten der Schrifft, wi ihm Gott zugesaget, beigefüget« (KP I, 202) ${ }^{25}$ Die drei Propheten, oder besser gesagt: ihre Visionen, werden also verstanden als Verlängerung der Heiligen Schrift - und damit als Übergangsfiguren für das dritte Zeitalter: "Nun kommet offen [...] der Göttliche Endzwekk aller solcher Verborgentheiten, das ewige Reich Jesuels, als es mit der Prophetin Christina am 24 October 1628 so geheim bezeichnet « (KP II, 271). ${ }^{26}$

In gewissem Sinne sagen die heutigen Propheten, gemäß Kuhlmann, nichts anderes als die Propheten der Heiligen Schrift, sie stehen lediglich im alchemo-theologischen Prozess der Sichtbarmachung des Unsichtbaren etwas näher an der Jetztzeit: "Forschet nach, ihr Kinder Gottes, in den heutigen Propheten, und si sind, di euch klährer hirvon zeugen. | Forschet in den Propheten der H. Schrift, und ihr findet darinn heimlicher, was in disen klährer abgehandelt." (KP I, 47) - auch hier also ein Prozess der Verwandlung vom Heimlichen zum Klaren.

Über den Kühlfürsten und -fürstinnen steht schließlich der »Kühlmonarch« (KP II, 178, V. 14267); in seiner Funktion nur dem "Hauptmonarch[en]《 Jesus Christus untergeordnet (KP II, 59, V. 10743). Und dieser Kühlmonarch ist niemand Geringeres als "Sanct Quirin" oder "Sanct Kühlmann" (KP I,

24 Hierzu Walter Dietze, Kuhlmann, S. 144-152; Wilhelm Schmidt-Biggemann, Apokalypse, S. 167-187 (allgemein), S. 192-195 (Kühlmanns Verhältnis zu Comenius und den von ihm veröffentlichten Texten).

25 Ich zitiere hier wie im Folgenden nach der Ausgabe Quirinus Kuhlmann, Der Kühlpsalter, hg. von Robert L. Beare, Tübingen 1971.

26 Vil. zur Entstehung und Verwendung des Begriffs »Jesuel« bei Kuhlmann Walter Dietze, Kuhlmann, S. 245; Wilhelm Schmidt-Biggemann, Apokalpyse, S. 218; Sibylle Rusterholz, Kuhlmann, S. 260. 
202); ein Heiliger also, doch nicht nur das. Er versteht sich, wie er in einem Brief an seinen jetzigen Gegner (aber früheren Vertrauten und Lehrer), den in Amsterdam lebenden Arndt- und Böhme-Anhänger Friedrich Breckling (1629-1711), ${ }^{27}$ schreibt, auch in einem politischen Sinne als eine Auctoritas: ${ }^{28}$ "Di Stunde ist da, daß die falschen figuren müssen fallen, weil meine figur ins wesen dringet, und di könige des falschen virreichs in ihren selbstfallenden Saulen feuerlichstprobiret werden« (KP II, 287; recte: 387). Ganz ähnlich formuliert Kuhlmann das auch im Haupttext des Kühlpsalter:

Von nun an weichen di Kaiser den Kühlmännern, di Virreiche der Kühlmonarchi im Wunder der Wunder aller Wunder. Von nun an haben alle Reichswechselungen sich endlich ausgewechselt; alle Reichssonnen sich endlich ausgesonnet, weil di Stunde da, von welcher der Prophet spricht: Es wird eine solche Unruhe unter den Sternen entstehen, das man auch vermeinen wird, es werde der Himmel zu grunde gehen, und di Erde nicht bleiben können. [...]. Das Gesätz währet mit allen Propheten bis auf Johannem: Johannes mit allen Aposteln bis auf den Kühlbund der Kühlordnung; mit welchem di heiligen Propheten und Aposteln vollkommen zugesigelt und entsigelt werden. (KP II, 102)

Bemerkenswert ist die politische Dimension dieser Vision: Als im "Centrum" des Anbruchs der Gottesherrschaft Christi auf Erden stehend ist die Kühlmonarchie der 'Rechtsnachfolger der in Dan 2 erwähnten, vier aufeinander folgenden Reiche (zu verstehen als letzte Form einer Translatio imperii). Aber natürlich handelt es sich im Grunde bei dem hier formulierten Quintomonarchismus ${ }^{29}$ der besagt, dass nach dem Vergehen der vier weltlichen Reiche ein göttliches entstehen wird, um eine politische Theologie: Angesprochen wird das Alte Testament, d.h. "alle[ ] Propheten", und die Überführung deren Prophetien in das Neue Testament durch die Übergangsfigur "Johannes mit allen Aposteln« (mit Rekurs auf Mt 11,13). Der Kühlbund ist hingegen schon in einer dritten Zeitordnung, deren Übergang ausdrücklich benannt wird: »bis auf den Kühlbund der Kühlordnung«. Diese dritte Zeitordnung ist durch die - explizit angesprochene - Zeitenwende ("Es wird eine solche Unruhe unter den Sternen entstehen «; Rekurs auf Offb 6,12-

${ }^{27} \mathrm{Zu}$ diesem Verhältnis Walter Dietze, Kuhlmann, S. 113-116; Wilhelm SchmidtBiggemann, Apokalypse, S. 192.

28 Vgl. zu diesem Begriff Giorgio Agamben, Ausnahmezustand, übers. von Ulrich Müller-Schöll, Frankfurt a.M. 2006, S. 88-106.

29 Zu Kuhlmanns Verhältnis zu den Quintomonarchisten, vgl. Walter Dietze, Kuhlmann, 135f., und Wilhelm Schmidt-Biggemann, Apokalypse, S. 197-200. Zum Quintmonarchismus allgemein, vgl., allerdings auf England konzentriert, Bernard S. Capp, Fifth Monarchy Men. A Study in Seventeenth-Century English Millenarianism, London 1972. 
14) deutlich von den ersten beiden unterschieden. Daher sind die (Aussagen aller) Vertreter des Alten Testaments (»Propheten«) und des zweiten (»Johannes mit allen Aposteln«) »zugesigelt«. Zugleich wird aber im dritten Zeitalter des Kühlbundes auch all das wieder, nur auf höherem, klarerem Niveau, aufgenommen, was in den Zeitaltern des Vaters und des Sohnes gesagt wurde. Daher werden die Propheten und Apostel auch wieder »entsigelt« (Rekurs auf Offb 5-8: das Buch mit sieben Siegeln).

Der Kühlmonarch scheint also der Prophet und Eröffner des neuen Zeitalters, auf den die anderen Propheten, die weniger klaren der Heiligen Schrift und die schon klareren der neueren Zeit, aus unter-, oder genauer: vorgeordneter Position verweisen. Insofern ist es alles andere als verwunderlich, dass sich auch Kuhlmann mit Elia - auch und besonders als Artista - beschäftigt; nicht zuletzt, weil er glaubt, dass sein Vorname, Quirinus, den des Elia bzw. Elias in sich schließt. ${ }^{30}$

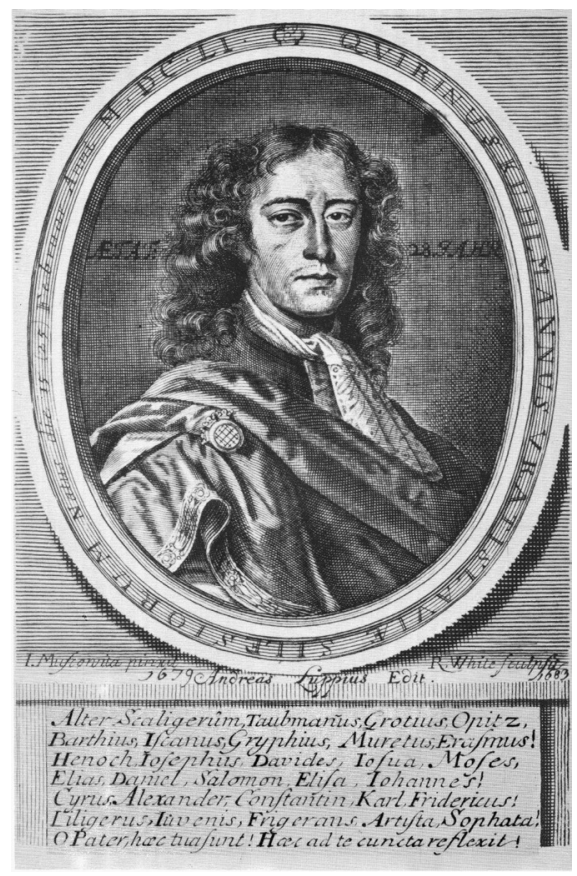

Abb. 1 Robert White (Graveur), Kupferstich Quirinus Kuhlmann ${ }^{31}$

30 Hierzu Walter Dietze, Kuhlmann, S. 150.

31 Entnommen: Quirinus Kuhlmann, Der Kühlpsalter, hg. von Robert L. Beare, Tübingen 1971, Bd. I, nach S. XXIII. 
Genauer gesagt sieht sich Kuhlmann in der Nachfolge, ja in einer Art von Identifikation von Elias Artista. Die Subscriptio seines Porträts beispielsweise ist in mehrere Namensserien geteilt.

Die erste Serie enthält Namen von Autoren, die Philologen, verstanden als Liebhaber des Wortes Gottes, und Dichter in einer Person waren. Zu denen zählt Kuhlmann u.a. Opitz, Gryphius und eben auch sich selbst. Die zweite Gruppe nennt Propheten des Alten Testaments, in deren Tradition sich Kuhlmann ebenfalls verortet. Gleiches gilt für die dritte Serie, welche die heilsgeschichtlichen Könige nennt, deren Abschluss Kuhlmann als Quintomonarch darstellt. Wichtig in diesem Zusammenhang ist nun die vierte Gruppe, in der es um chiliastische Selbstzuschreibungen Kuhlmanns geht, von denen eine, wie nicht anders zu erwarten, der "Artista ist.

Eng verbunden ist der "Artista", auch dieser Begriff findet sich in der vierten Serie, mit dem »Liliger«. Dies spielt auf die Rosen und Lilien, die bei Böhme die Symbole der Vollendung im Millenium darstellen. ${ }^{32}$ Dementsprechend sieht sich Kuhlmann, wie er im Kühlpsalter schreibt, "mit der Regenbogichten Naturlilge des Elias Artisten " (KP I, 58) geschmückt und fühlt sich, von seiner Seherkraft her, "unter dem gantzem Centrum Elias Artistens" (KP I, 296). "Centrum« ist, wie man hinzufügen muss, ebenfalls ein Lieblingsbegriff Böhmes, den Kuhlmann hier übernimmt. ${ }^{33}$ Es wird deutlich, dass Kuhlmann über den Technik-Propheten Elia ins Zentrum des göttlichen Wesens und damit an das Ende aller Zeiten zu greifen vermeint. Er fühlt sich also, zu diesem Zeitpunkt, mit Elias Artista vereint, von ihm gestärkt, aber er ist nicht Elias Artista. Noch nicht.

Elia wird bei Kuhlmann, im Sinne des oben rekonstruierten Joachimismus, in zweifacher Funktion verwendet, einerseits als Prophet des Alten Testaments und andererseits als Eröffner des Neuen Zeitalters. Im ersten Falle wahrt Kuhlmann eine klare Distanz zwischen sich und dem Propheten; z.B. sieht sich der Verfasser bei seiner Reise im "geiste schon mit Mosen und Elien " (KP II, 155). ${ }^{34}$ Man muss dazu wissen, dass Moses, alternierend mit Henoch, seit der Patristik neben Elias der entscheidende Zeuge für den Anbruch des Endes der Zeiten ist, zu denen Johannes hinzutreten kann, aber

32 Hierzu Wilhelm Schmidt-Biggemann, Apokalypse, S. 173-177; S. 212-214; S. 223f.; Harald Haferland, Heilsbedeutung, S. $153 f$.

33 Vgl. ebd.

34 Vielleicht eine Anspielung auf Mk 9,4, wo diese beiden Jesus bei seiner Verklärung erscheinen. 
nicht muss. ${ }^{35}$ Dementsprechend wird zur figuralen Aneignung der beiden Figuren aufgerufen: »Wo ist [...] Des Moses neue streng? Eliens wunderthat?" (KP II, 228, VV. 15814-15815) Aber diese Aneignung scheint noch nicht stattgefunden zu haben.

Es gibt aber auch Zeichen, aus denen sich schließen lässt, dass sich der alttestamentliche Prophet bereits im Übergang vom zweiten zum dritten Zeitalter befindet. Elia kann nämlich, auch als Prophet des Alten Testaments, bereits die Rolle des Verkünders und Eröffners des dritten Status einnehmen: "Denn der Anfang hat das Ende, und das Ende den Anfang funden, das ist, di Erndzeit der Welt, nachdem bald sechsjahrtausende gesäet, ist herbeigenahet, und mus das Unkraut nunmehro aus allen Völkern gesammelt werden, um mit dem Eliasfeuer es zuverbrennen«, heißt es in der Vorrede zum dritten Buch (KP I, 94).

Das Feuer in diesem Zitat entspricht dem, das Gott auf dem Berg Karmel - anstelle des Gottes Baal - auf das von Elia gerichtete Opfertier herniederbrennen lässt (1 Kön 18,38). Zum Verständnis des Zitats gilt sich weiterhin zu gewärtigen, dass sich Kuhlmann hier der im alchemischen Kontext üblichen Metapher vom Ende der Tage als der "großen ärnt« bedient. ${ }^{36}$ Die Metapher ist in gewissem Sinne dreigliedrig: Sie läuft über eine Parallelisierung des alchemischen Prozesses mit der Reife- und Erntezeit der Natur (wobei der alchemische Prozess grundsätzlich deren Beschleunigung darstellt). ${ }^{37}$ Dieses Ernteverständnis wird nun von der irdisch-alchemischen auf die alchemotheologische, genauer: apokalyptische, Ebene gehoben: das Ende der Zeit als großen Ernte. Zugleich behält dieses theologische Feuer noch eine alchemisch-technische Bedeutung: als Verbrennungsprozess, in dem eine, wie es z.B. bei Adam von Bodenstein heißt, "scheidung deß guten vom bösen« stattfindet. ${ }^{38}$

Dieses grundsätzlich alttestamentliche Bild wird jetzt auf die Unkrautausrottung im Sinne von Mt. 13, 24ff. (Gleichnis vom Unkraut unter dem Weizen) übertragen - und zwar durch das Feuer, das Elia darstellt bzw. in Brand gesetzt hat. Es handelt sich also bei Elia (bzw. dessen Handlungen) um eine "straff" gegenüber den Ungläubigen (KP I, 161, V. 4579). Das

35 Vgl. hierzu die immer noch unentbehrlichen quellenreichen Ausführungen in Wilhelm Bousset, Der Antichrist in der Überlieferung des Judentums, des neuen Testaments und der alten Kirche. Ein Beitrag zur Auslegung der Apocalypse, Göttingen 1895, S. 134-139. Paracelsus, De generatione et destructione regnorum, SW II/2, S. 149.

37 Vgl. hierzu Vf., Nachahmung, S. 113-131.

38 Bodenstein, Widmungsvorrede an den Magistrat der Stadt Mülhausen, 24.8.1562, zu: Paracelsus: Paramirum, CP I, 272. 
Unkraut, das verbrannt wird, ist das Sündenbabel. Zu einem Zeitpunkt, da »di Erndte naht!«, tut der »thumme[ ] Hauf nämlich genau das Falsche: Er handelt wie von "Bel verblendt" und erntet daher nur "Babelsaat", die wiederum in "Elias feur" aufgehen soll bzw. wird (KP I, 210, VV. 5941-5947). Alttestamentlich gesehen, wird hier der Prophet Elia als Bekämpfer des Baalskultes (durch "Bel verblendet«) über die alchemische Figur der ringförmigen Verschließung von Anfang und Ende ${ }^{39}$ mit seiner Wiederkehr und Eröffnung der Gnadenzeit Christi (»di Erndte naht«) zusammengedacht. Elia, als Eröffner des Gnadenreichs, wird alles Falsche des zweiten Status vernichten, wie er, als Prophet des Alten Testaments, alles Falsche des Baalskultes im ersten Status dem Feuer überantwortet hat.

Diese Überschneidung von alttestamentlicher und neutestamentlicher bzw. endzeitlicher Elia-Gestalt setzt sich auch im weiteren Verlauf des Kühlpsalters fort: "Das Hohpropheten-amt im Centrum ist vollendt: | Elias kunfft ist da zum feuerwunder. | Das Königsamt im Centrum kommt herbei!« (KP II, 284, VV. 18124-18126). Elia ist, diesem Zitat zufolge, Hoch-Prophet (des Alten Testaments) und derjenige, der wiederkehrt zum »feuerwunder« des Übergangs vom zweiten ins dritte himmlische Saeculum.

Schon am Ende des dritten Buchs des Kühlpsalters kommt es zur ersten Identifizierung des Verfassers mit Elia. Der 53. Kühlpsalm formuliert ein »lid« zum "lobpreis fehova fesus" (KP I, 233), das für jeden Tag der Woche und für jede Tageszeit dieses Tags einen eigenen Text bereitstellt. Am Ende, also am "Sonnabend« (die Wochenrechnung beginnt mit dem Sonntag), wird ein Lied für den Abend geboten, dessen Strophen mit den Buchstaben des Alphabets beginnen. Am Ende (des Liedes und des gesamten Psalms), also in der Strophe mit dem Buchstaben Z, ist Kuhlmann bei dem angelangt, auf das er hinsteuert, auf das "Zeitende" (KP I, 284, V. 7000). Beschrieben wird, was im ganzen Psalm, ja im ganzen Kühlpsalter schon vorbereitet wurde, nämlich eine Art von Unio mystica, die den Weg von außen nach innen sucht: »(Von aussen nur am zeitgetümmel;) | Inwendig schon an Gottesschos | Und alles euserlichen los" (ebd.).

Und dieser Zustand wird jetzt alttestamentlich (bzw. in neutestamentlicher Interpretation des Alten Testaments) mit einer dezenten Identifizierung des Verfassers mit den zwei großen Entrückten des Alten Testaments, nämlich Henoch (1 Mos 5,24) und, wem sonst, Elia (2 Kön 2,11) beschrieben:

39 Vgl. Robert Fludd, Anatomiae amphitheatrum effigie triplici, more et conditione varia designatum, Frankfurt a.M. 1623, S. 314f.: »Deus est omne quod est. Ab eo procedunt omnia \& iterum in eum revertuntur«; ’Gott ist alles, was ist. Von ihm geht alles aus und kehrt auch wieder in ihn zurück`. 
Sei aussen unter den vir winden:

Inwendig bei den Gotteskinden,

Bei Jesus Christ im Paradeis

Auf Henochs und Elias Reis.

Nihm, Jesus, mich in deine hände

In solchem Geist an meinem ENDE. (KP I, 284, VV. 7004-7009)

Wie oben bereits ausgeführt, ist Henoch, alternierend mit Moses, neben Elia der entscheidende Zeuge für die Anzeige des Endes der Zeiten. Doch mit "Ende" ist bei Kuhlmann nicht nur das Zeitenende beschrieben, sondern zugleich das Ende des Lieds und des Psalms - aber nicht des Kühlpsalters, der nach dieser Andeutung des Endes noch über 10000 Verse (die Prosaeinschübe nicht mitgerechnet) für den Leser bereithält. Wenn man also davon ausgeht, dass Kuhlmann das Zeitenende und im gewissen Sinne sein Ende in Gott herbeischreiben möchte, dann sind wir hier noch ganz am Anfang dieses Endes.

Das zeigt sich auch daran, dass Kuhlmann und Elia, trotz aller Ähnlichkeiten, bis jetzt noch zwei unterschiedliche Personen sind. Aktuell geht es dem Autor des Kühlpsalters darum, die Aufgabe, die Elia gestiftet hat, zu übernehmen: "Nihm munter an Elias werk! - auch hier in der Rolle des Vernichters des Falschen: "Verzehre si mit Gottesflammen!« (KP I, 284f., VV. 8266; 8268). Ähnlich in KP II, 4: „Gehe hin [...] in der stärke Eliens, schlage deine und meine Feinde darnider". Wie leicht zu sehen ist, bezieht sich diese Form der Imitation noch vorderhand auf den alttestamentlichen Propheten und Bekämpfer des Baalskults und nicht so sehr auf denjenigen, der das Reich Christi ankündigt und eröffnet.

Die Vorrede zum fünften Buch macht nun deutlich, dass Kuhlmann sich jetzt als Elia, verstanden als Wiederkehrer und Eröffner des Reiches Christi, sieht. Diese Apostrophierung ist insofern etwas kompliziert, als Kuhlmann zugleich die Figur zu sein meint, welche die Kühlpropheten Kotter, Drabitz und Poniatovska vorausgesagt haben: "Diser hirstehende, ruffet der Prophet Drabitz, ist von Gott selbsten erwählek, heißt es von Kuhlmann über Kuhlmann. Und »dieser hirstehende« ist auch "Elias», aber nicht nur verstanden als der Prophet des Alten Testaments, sondern "diser Elias", also verstanden als derjenige, der als Elia wiederkehrt und das Reich Gottes ankündigt (KP II, $5)$.

Möglich wird dieses Spiel mit der Figur des Elias (Figur wiederum im Sinne Auerbachs verstanden) durch, ich habe das oben bereits erwähnt, Bibelstellen wie Mal 3,23f., wo zu lesen ist, dass Elia als Verkünder einer Neuen Zeit wiederkommen wird - und natürlich auch durch das Neue Testament, 
in dem seine Wiederkunft, z.B. in der Figur Johannes (Joh 1,21 u.ö.), beschrieben wird. Dass Kuhlmann in diesen Kategorien der Aufnahme des Alten im Neuen Testament denkt, wird z.B. auch aus Formulierungen deutlich, die besagen, »das Moses Petrisirt, | Und das Johann Eliens feur heut führt« (KP II, 74, VV. 11217-11218).

Die figurale Überblendbarkeit der Figur Elia durch Johannes ermöglicht Kuhlmann nun deren Öffnung und Neubesetzung, aber eben nicht mehr in Bezug auf das Neue Testament und den neuen Bund, sondern in Bezug auf den dritten Status. In ihm ist es nicht mehr Johannes, sondern niemand Geringeres als Kuhlmann, der in der Figura des Elia auftreten kann.

\section{III. Über Elia hinaus: Der Sohn von Gottes Sohn}

In gewissem Sinne ist Kuhlmann auch schon über Elia hinaus, da er nicht nur, wie dieser, "ein Mann [...] nach dem hertzen Gottes« ist und daher Prophetie und Eröffnung des Neuen Zeitalters in die Wege leiten kann. Vielmehr ist er auch derjenige, der, gemäß der "Prophetin Christina", "regiren " wird, "wi« Gott es "haben wil" (KP II, 5). Er übernimmt also, als Übergangsfigur, nicht nur die Rolle des Propheten, sondern sieht sich - auch hier muss man sagen: noch - in der Stellvertreterschaft Gottes, dessen Herrschaft am Zeitenende anbricht.

Dass Kuhlmann über Elia hinaus will und muss, wird schon aus der Terminologie deutlich. Oben wurde ausgeführt, dass die Verbrennung ein wichtiges Merkmal für Elia ist, wie gesagt mit einer klaren alchemischen Denotation dieses Begriffs (die Scheidung des Guten vom Falschen im alchemotheologisch zu verstehenden Verbrennungsprozess), so zu lesen z.B. im 42. Kühlpsalm: "Noch Henoch würkt mit Mosen und Elien" - hier wird wiederum das endzeitliche Zeugenpaar aufgerufen - "Bis Babel wird im grundfeur feurig glühen" (KP I, 178, VV. 5104-5105).

Kuhlmann hingegen ist der Kühlmonarch, dessen Kühlung zu dem Zeitpunkt einsetzt, da Elia das Falsche verbrannt hat. Durch diese Gegenüberstellung kommt nun das Verbrennen in die semantische Nähe des »Satan[s]《; der "gibt dem Kohlmann kohlen«, wohingegen "Gott durch den Kühlmann kühlt« (KP I, 228, VV. 6441-6442). Diese Reihenfolge entspricht grundsätzlich der biblischen Geschichte: Erst sendet Gott, auf Geheiß des Elia, Feuer, dann den kühlenden Regen, auf den die Israeliten in der Dürre so lange gewartet haben. Über dieses Szenario legt Kuhlmann nun die Assoziation 
Feuer/Hölle und nutzt so die Feuer/Wasser-Dichotomie zur Absetzung bzw. Überwindung von Elia.

Dass Elia, als Artista oder auch nur als reiner Prophet, für Kühlmann letztlich nicht mehr als eine Durchgangsstation ist, wird alttestamentlich wie neutestamentlich ausgeführt. Kuhlmann ist es nämlich durchaus bewusst, dass "Elia«, als alttestamentlicher Prophet, nicht der Letzte ist und in "Elisa « - mit denen zweien wandelt er durch Bethlehem und Jericho - seinen Nachfolger hat (KP II, 166). Gleiches gilt aus neutestamentlicher Perspektive bzw. für den Übergang zum dritten Gnadenreich, bei dem Kuhlmann selbst ins Spiel kommt: "Elias zeit ist hin durch zweiundvirtzig Monden. | Nun komm ich kräfftig an mit Jesus Millionen" (KP II, 62, VV. 10847-10848). Und ganz deutlich wird die Überwindung des Elia im letzten, dem 117. Kühlpsalm: Mittlerweile erwägt Kuhlmann, dass Gott »von mir als neuen Adam sprach[] ] - und dementsprechend auch "mich vorzog[ ] dem Moses und Elias" (KP II, 319, VV. 19789; 19801); beide, wie gesagt, neben Henoch die entscheidenden Zeugen der Endzeit.

Der Grund für die Überbietung von Elia ist der, dass Kuhlmann sich jetzt mehr und mehr in die Rolle des eigentlichen Herrschers des dritten Zeitalters hineinwachsen sieht. Denn dass dieses angebrochen ist (oder zumindest im Anbruch ist), daran besteht ab dem achten und letzten Kühlpsalter keinerlei Zweifel mehr: »Von nun an wird das geheimnis des Allmächtigen vollendet werden, weil sein göttlicher termin erschinen! Von nun an werden wir mit himmlischen augen nachsehen den Patriarchen, Propheten, Aposteln, Kühlmännern, weil das himmlische Reich sich bildet!« (KP II, 271) Es ist in diesem Zusammenhang sehr hilfreich, sich die Selbsttitulierungen in den Vorreden der jeweiligen Kühlpsalter-Bücher anzusehen. Die des achten Buchs unterschreibt Kuhlmann nämlich, nachdem er das erste noch demütiglich als »ein Christ« (KP I, 4) und die weiteren, schon souveräner, aber noch in der christlichen Zeit verbleibend, als ein "geruffener Printz Gottes der Israeliten, Christen, Jesueliten« (KP II, 103) signiert hatte, bereits als Gottes-Sohnessohn: "Der Sohn des Sohnes Gottes Jesu Christi und Printz, Prophet, Prister des ewigen erlösten Königreiches Jesuels« (KP II, 274). ${ }^{40}$ Daraus erhellt: Auch der Übergang vom Altem zum Neuen Testament (Jesus als Gottes Sohn) wiederholt sich noch einmal im Übergang vom Neuem Testament oder zweiten Säkulum ins anbrechende Gnadenreich: Gottes Sohn hat seinerseits einen Sohn; und als dieser Sohnessohn Gottes fungiert

40 Vgl. zur Entwicklung der Sohnessohn-Theorie bei Kuhlmann, Walter Dietze, Kuhlmann, S. $245 \mathrm{f}$. 
niemand anderes als der, der sich an Elia abgearbeitet, sich ihm einverleibt und ihn schließlich hinter sich gelassen hat: nämlich Kuhlmann selbst. ${ }^{41}$

\section{Die Schreibart des Kühlpsalters}

Was für die Kühlmonarchie gilt, gilt auch und besonders für den Kühlpsalter, sozusagen als dessen schriftliche Ausbuchstabierung: "Der Kühlpsalter ist die Erfüllung aller Kühlpropheten, Kühlweisen, Kühlschrifftgelährten, und beginnet daselbst, wo der Prophet Drabitz aufhöret«, heißt es im »Inhalt des Kühlpsalters«, einer Art Erklärung des gesamten Werks (KP I, 313).

Damit geht Kuhlmann noch einmal über das hinaus, was er zu Beginn des Kühlpsalters geschrieben hatte: "Di Kühlungszeit, davon alle Propheten von anbeginne der Welt gesprochen, knospet albereit seine lilien- und Rosenknospen« (KP I, 203). Lilie und Rose sind, gemäß Böhme (s.o.), Symbole der Vollendung im Millennium. Während zu Beginn des Kühlpsalters noch eine gewisse Prozessualität der Erfüllung angenommen wird (die Knospen, die noch zur Reife kommen müssen), wird in der Zusammenfassung davon ausgegangen, dass das dritte Zeitalter bereits angebrochen ist.

Damit ist besagt, dass der Kühlpsalter nicht einfach nur die Kühlmonarchie beschreibt. Vielmehr ist er ihre schriftliche Form. Wenn die neueren Propheten klarer sind als die alten, da deren Visionen, wie oben ausgeführt, noch weitgehend geheim waren, dann ist der Kühlpsalter die klarste Form. In ihm ist alles Wissen offenbar geworden. Dass der Kühlpsalter nicht einfach eine Verlängerung der älteren Prophezeiungen ist (»beginnet daselbst, wo der Prophet Drabitz aufhöret«), sondern die schriftliche Form ihrer Realisierung, darauf verweist der Begriff der »Erfüllung«.

Es handelt sich bei der "Erfüllung« um einen intrikaten Zwischen-Begriff, der zwischen den der Prophezeiung und den des Eintretens (der Prophezeiung) tritt. Für Kuhlmann steht nämlich zwischen den bisherigen Prophezeiungen, die immer klarer wurden, und ihrem tatsächlichen, realen Eintreten, noch eine Zwischenstufe, nämlich die Erfüllung im Sinne von: Klarwerden aller Prophezeiungen. Und erst wenn diese schriftliche Form der Erfüllung eingetreten ist, kann die wirkliche Erfüllung (im Sinne von: Eintreten) erfolgen.

41 Vgl. zur Selbststilisierung Kuhlmanns, Walter Dietze, Kuhlmann, S. 140f. 
Damit beschreibt Kuhlmann eine wechselseitige Form der Abhängigkeit der älteren Prophetien zu seinen eigenen im Kühlpsalter, auf den alles zuläuft: "Darum mag nimand verstehen di Kühlpropheten ohne den Kühlpsalter, noch den Kühlpsalter ohne di Kühlpropheten, weil sie alle von mir zeugen" (KP I, 314). Diese zeitverkehrte Zeugenschaft gilt übrigens nicht nur für die drei Comenischen Philosophen, sondern auch für den Spiritus Rector seiner Schreibweise, Jacob Böhme: »da selbst mein Böhme jauchtzet, weil er zu meinem zeugnisse geschriben, und den grossen Character, der alleine mein eigen, empfangen« (KP I, 315f.).

Auf Basis der hier geäußerten Vorstellungen kommt Kuhlmann nun zu dem bereits rekonstruierten Gedanken, dass der Elias Artista alles buchgestützte Wissen unnütz machen wird, weil nun das Wissen offenbar ist: "Was in allen büchern stükkweise ist gegeben, haben wir in Gotteskrafft im vollem Centrum, und schreiben wir nach unser Erkäntnis, zur ehre unsers Schöpffers, Erlösers, Heiligmachers in einem solchen stil als di zeit fodert, welche anstehet« (KP I, 315).

Bemerkenswerterweise (man könnte auch sagen: notwendigerweise) geht Kuhlmann dem Gedanken aus dem Weg, dass mit dem Zeitpunkt, da alles Wissen der Welt nicht mehr "stükkweise", sondern ganz und in Klarheit gegeben ist, eigentlich gar keine Bücher mehr notwendig sind. Denn das war ja der, über Böhme transportierte, paracelsisch-paracelsistische Gedanke des Elias Artista: Bis zu seiner Ankunft, so die Paracelsisten, schreiben alle Autoren Bücher, die zwar klarer sind als alles, was vorher geschrieben wurde, aber die Prozessualität von Schreiben, Auslegen, Neu-Schreiben verhindert, dass im Medium Buch alle Geheimnisse vollständig offenbar werden. Jedes Buch legt das letzte aus und muss wieder ausgelegt werden. Erst der Artista macht diesem Prozess der stetigen Perfektibilität des buchgestützten Wissens ein Ende - indem er das buchgestützte Wissen als solches überflüssig macht.

Würde Kuhlmann aber diesen Gedanken vollends mitgehen, würde er sich, ich habe es einleitend erwähnt, in einen performativen Selbstwiderspruch bringen, da er - seinem speziellen Begriff der Erfüllung der Prophezeiungen folgend - nur im Medium Buch das Ende der Bücher verkünden kann. Also muss sein Argument anders lauten, nämlich dass dieses Buch das letzte sei und sich daher grundlegend unterscheide von allen anderen vorher veröffentlichten Büchern; und zwar durch den (dieser Begriff fällt ja im Zitat wörtlich) "stil«.

Es geht also, Kuhlmann zufolge, nicht um das Ende der Bücher, sondern um einen radikalen Wandel in Stil und Schreibweise. Das sind die Begriffe, 
die der Kühlpsalter für seine Selbstbeschreibung verwendet. Dieses Buch der Bücher ist, wie man hinzufügen muss, als ein drittes Testament ${ }^{42}$ gedacht, wie folgendes Zitat deutlich macht: »Sihe nun den ersten Ekkstein zur neuen Schrifft und Kühlungsbunde in disem Kühlpsalter nach allen Propheten aller zeiten, und erwarte dessen gemeinmachung mit Wundern unter allen Völkern« (KP II, 169).

Der Text, der alle Prophezeiungen aller Zeiten noch einmal in sich in einem neuen Dokument fasst und zugleich einen neuen, d.h. dritten, Bund (den »kühlungsbunde«) mit allen Menschen schließt, ist also nichts weniger als eine "neue Schrift« oder ein neues Neues Testament. ${ }^{43}$ Dass Kuhlmann in diesem Zusammenhang den Begriff des Ecksteins verwendet - Eckstein ist ja eigentlich Jesus selbst ${ }^{44}$ - belegt, dass er sich hier bei der Abfassung dieser neuen, neuen Schrift zum neuen, neuen Bund im Vollgefühl göttlicher Kraft bewegt.

Eng verknüpft mit der Frage des Stils ist die Frage, wer eigentlich den Kühlpsalter schreibt? Wie Böhme bemüht auch Kuhlmann das Paradox mystischen Schreibens: „Wi vilmahls jauchtzeten di himmlischen herscharen in den inneren Welten, wann wir in unserm einfältigen glauben getriben, schriben" (KP I, 314), heißt es im 36. Kühlpsalm. ${ }^{45}$ Einerseits, so lässt sich daraus schließen, finden wir im Kühlpsalter ein riesengroßes Autoren-Ich vor, das sozusagen, um mit Angelus Silesius zu sprechen, noch »über Gott « ${ }^{46}$

42 Vgl. auch Wilhelm Schmidt-Biggemann, Apokalypse, S. 194f. Dass der Kühlungsbund den alten und neuen (als dritten) Bund ablöst, beschreibt auch Sibylle Rusterholz, Klarlichtige Dunkelheiten, S. 226.

43 Vgl. auch Wilhelm Schmidt-Biggemann, Apokalypse, S. 194.

44 Im Ps 118, 22 heißt es: "Der Stein den die Bawleute verwerffen / Jst zum Eckstein worden«. Diese Passage wird bei Matthäus (Mt 21,42), Markus (Mk 12,10) und Lukas (Lk 20,17) für das Gespräch zwischen Jesus und den Hohepriestern aufgegriffen. Zentral ist weiterhin die Apostelgeschichte Kapitel vier, innerhalb deren Petrus und Johannes nicht nur auf Ps 118, sondern auch auf Jesu Aufgreifen dieses Psalms in den Evangelien rekurrieren. Petrus, "vol des heiligen Geists", und Johannes disputieren hier ebenfalls mit den "Priester[n] [...] des Tempels" und sprechen, nun schon das Perfekt verwendend, von Christus als dem Gründer ihrer Kirche: "Das ist der Stein / von euch Bawleuten verworffen / der zum Eckstein worden ist. Vnd ist in keinem anderen Heil / ist auch kein ander Name den Menschen gegeben I darinneb wir sollen selig werden " (Apg 4,1; 8; 11f.; alle Zitate nach der Luther-Bibel von 1545 nach Martin Luther, Biblia, das ist, Die gantze Heilige Schrifft Deutsch, hg. von Hans Volz, 2 Bde., Darmstadt 1972 [= ND der Ausgabe Wittenberg 1545]).

45 Zum Zusammenhang von mystischem und chiliastischem Denken allgemein und bei Kuhlmann im Besonderen, vgl. Alan Menhennet, ,Wir tunken ein unseren Kil in die ewigkeitr. Quirinus Kuhlmann's experience of eternity, in: Forum for modern Language Studies 27 (1991), S. 159-168, hier S. 160f.

46 „Man muß noch über Gott« betitelt Scheffler das Epigramm I/7 und führt in Vers vier aus: "Jch muß noch über Gott in eine wüste ziehn" (Angelus Silesius [d.i. Johannes Scheff- 
ist, weil das von ihm eröffnete Zeitalter weiter als dessen zwei Testamente ist. Andererseits, und das genau macht das Paradox mystischen Schreibens aus, ist dieses Ich nichts, weil der Text von Gott verfasst wurde und nur via Glauben und Feder in der Hand Kuhlmanns niedergeschrieben wurde: »in unserm einfältigen glauben getriben« (s.o.).

Auf den Gedanken, dass es der reine Glaube ist, der das Wissen und das wissensgestützte Schreiben überflüssig und unnütz machen wird, kommt Kuhlmann immer wieder zurück: "Ich ging im tribe des grossen Centrums als ein kind voller einfalt, als alle dise selbstklugen zu anderen Werken vom grossen Werk mich abzogen, und bin doch endlich, ungeachtet aller hindernisse, durch das gesätzte zeitzil durchkommen« (KP I, 315). Der Kühlpsalter schreibt sich also nicht aus gelehrtem Wissen, sondern aus, Kuhlmann bezieht sich hier, wie viele seiner Zeitgenossen, auf Augustinus, ${ }^{47}$ kindlicher "einfalt" heraus - und damit anders als alle "anderen Werke«. Er ist nicht ein Werk unter vielen, sondern das "grosse Werk«; und dieses große Werk kann man nur schreiben, wenn man nicht mehr Ich ist, nicht mehr über individuelles Wissen verfügt, sondern sich vollständig »im tribe des grossen Centrums« treiben lässt.

Ort der nicht-verstandesmäßigen ${ }^{48}$ Übermittlung göttlicher Gedanken ist, wie in der paracelsischen Tradition üblich, das Gemüt: ${ }^{49}$ Hier entsteht die Sprache des Kühlpsalters; eine Sprache, über die Kuhlmann nicht zu verfügen behauptet: »Im übrigen werden alle Gesänge reden von dem Gemütte, daraus si geflossen, ob ich davon zureden ihnen gleich ni geboten" (KP I, 4). Das ist also der »stil« des neuen Neuen Testaments: schreiben und schreiben lassen. Wenn man diese Technik, die natürlich gerade keine Technik ist, beherrscht, kann man sich in einen Zustand jenseits der Zeit hineinschreiben: »Wundert euch nicht, Menschenkinder, dass unsere Schreibart di ge-

ler], Cherubinischer Wandersmann, Kritische Ausgabe, hg. von Louise Gnädinger, Stuttgart 1984, S. 28).

47 Augustinus, De vera religione, Abschnitt 65: »Unum certe quaerimus, quo simplicius nihil est. Ergo in simplicitate cordis quaeramus illum« - »Wir suchen ja das Eine, im Vergleich zu dem es nichts Einfacheres gibt. Suchen wir ses` also in der Einfachheit des Herzens" (Ders., Opera-Werke [lt.-dt.], hg. von Johannes Brachtendorf u.a., Paderborn u.a. 2002ff., Bd. VXVIII, S. 188f.). Vgl. hierzu auch Jörg Trelenberg, Das Prinzip 'Einheit‘ beim frühen Augustinus, Tübingen 2004, S. 83, und zur Wiederaufnahme der Gedankenfigur in Frühpietismus und Barockliteratur; Vf., Das Heuchelei-Dilemma, Moscheroschs 'Schergen-Teuffek als poetologische Ortsbestimmung satirischer Prosa, in: Nicola Kaminski u.a. (Hg.), Spielregeln barocker Prosa. Historische Konzepte und theoriefähige Texturen sungebundener Rede in der Literatur des 17. Jahrhunderts, Tübingen 2012, S. 43-68.

48 Zur Wissenschaftsfeindlichkeit Kuhlmanns, vgl. Walter Dietze, Kuhlmann, S. 130.

49 Vgl. hierzu Vf., Nachahmung, S. 45; S. 51; S. 88 u.ö. 
wöhnliche Schreibart aller gesandten von Nun an verlässet! Wir tunken ein unseren Kil in di Ewikeit, ein ewiger Kilmann, mit unserer Kronzahl der Engelswelt umleuchtet, und übertreffen so weit aller offenbahrungen, als di macht eines absoluten Printzens di macht aller seiner Unterthanen übertriffet« (KP I, 271).

Dieses Zitat fasst alle Gedanken, die vorher einzeln geschildert wurden, noch einmal zusammen: Die Kühlmonarchie (angesprochen durch den "Printz «, der seine "Unterhanen übertriffet«) wird auf das Thema der »offenbahrungen" umgelegt. Dementsprechend übertrifft deren schriftliche Form, der Kühlpsalter, alle anderen Texte zum Thema der Offenbarung, weil dieser Text die Auctoritas, die Souveränität für sie darstellt, unabhängig davon, ob sie vor ihm, nach ihm oder zeitgleich geschrieben wurden. In jedem Falle laufen die verschiedenen Offenbarungen von Elia über Johannes, Böhme und Drabitz auf Kuhlmann und den Kühlpsalter zu, weil in ihm Gott selbst spricht.

Und von diesem Zustand leitet sich die Schreibeweise des Kühlpsalters ab. In diesem Zustand muss man seinen Kiel - den Kuhlmann in seiner Privatetymologie in der Nähe des Kühlenden sieht ${ }^{50}$ - in das kühle Nass der Tinte eintauchen und schreiben. Aus diesen Gründen bedarf der Kühlpsalter einer eigenen "Schreibart", in der Gott durch den Kühlmann hindurchschreibt und damit diesem (und seinem Stil) alle göttlichen Souveränitätsrechte verleiht.

Für diese Schreibart des Über-Wissens gilt alles, was schon für die ersten beiden Testamente galt. Kuhlmann reklamiert also für sich eine Art von Sola Scriptura-Lehre: „Gegenwärtige Fünffzehngesänge werden nimals mit blossem lesen oder betrachten, sondern alleine in dem stande völlig verstanden werden, darinnen si geschriben" (KP I, 3). Luthers Sola Scriptura-Lehre besagt bekanntlich, dass "ausser der schrifft nichts soll gelert werden ${ }^{51}$ was in den Augen des Reformators deswegen möglich ist, da die Schrift eine innere wie äußere Klarheit besitzt. Bekanntlich setzt der Reformator gegen Erasmus von Rotterdam, der behauptet, dass es in der Bibel "adyta«, also »unzugängliche Stellen«, gäbe, ${ }^{52}$ die »claritas scripturae ${ }^{53}$ Diese Klarheit er-

50 Vgl. zum Begriffspaar Kiel/Kühlen bei Kuhlmann, Walter Dietze, Kuhlmann, S. 242.

51 Martin Luther, Antwort Bock Emser, Werke, Kritische Gesamtausgabe (Weimarer Ausgabe), Weimar 1883ff. Bd. VII, S. 662 (im Folgenden WA).

52 Erasmus von Rotterdam, Diatribe, in: Ders., Ausgewählte Schriften (lt.-dt.), hg. von Werner Welzig, übers. von Winfried Lesowsky u.a., 8 Bde., Darmstadt 1995, Bd. IV, S. $10 f$.

53 Martin Luther, De servo arbitrio, WA, Bd. XVIII, S. 609. 
fasse, so sein Argument, wer »den Geist Gottes habe« (»qui spiritum dei habet«).$^{54}$ Und genau das nimmt auch Kuhlmann für sich in Anspruch: Wer den Geist Gottes, sprich: Kuhlmanns, hat, der kann die Texte Kuhlmanns in ihrer vollkommenen Klarheit lesen, das heißt: Sie können »in dem stande völlig verstanden werden, darinnen si geschriben".

Der entscheidende Unterschied gegenüber der paracelsisch-paracelsistischen Elias-Artista-Vorstellung ist also der, dass sich Kuhlmann, als Autor des Kühlpsalters, nicht vollkommen aus der Zeit verabschiedet. Für die Paracelsisten wäre mit dem (von Kuhlmann behaupteten) Auftritt Elias' des Artisten ein Zustand jenseits der Zeit eingetreten. Daher würden dann alle in der Zeit befindlichen Prozesse der buchgestützten Wissensproduktion aufgehoben.

Nicht so für Kuhlmann. Für ihn beginnt mit der Vorstellung vom Dritten Testament nur eine neue Zeit, die natürlich nichts anderes als die »Kühlzeit« (KP II, 169 [Hervorh. MB]) sein kann (eine Anspielung auf Apg 3,20: "tempora refrigerï«, bei Luther: "erquickung «). ${ }^{55}$ Dieser Begriff ("Frigerans") befindet sich ebenfalls als chiliastische Selbstbeschreibung in der vierten Begriffsserie der Subscriptio seines Porträts (Abb. 1).

Die Konsequenz dieses Verbleibens in der (wenn auch neuen) Zeit ist, dass der Kühlpsalter wohl doch nicht das letzte Wort in dieser Sache darstellt: „Darum", heißt es im Inhalt des Kühlpsalters, »übergebe ich euch dis Buch, als ein kleinoth und das beste von meinem, in welchem haupttiffen nach der inneren und euseren welt; dessen Worte wichtig und samen sind, aus welchem ein Baum leiblich und sichtbar wächset durch das Paradeis in di Ewigkeit, wie in allen Propheten geweissaget " (KP I, 316).

Kuhlmann bleibt zwar dabei, dass in seinem Buch alles steht, wie es »in allen Propheten geweissaget«, bemüht also wieder die Gedankenfigur der literarischen Erfüllung der Prophetien. Gleichzeitig behauptet er, dass sein Buch sich nicht endgültig aus der zeitlichen Prozessualität der Wissensproduktion und -reproduktion befreien kann. Das Buch ist noch nicht in der "Ewigkeit«, sondern nur Samen, aus dem erst das erschriebene bzw. zu erschreibende Jenseits der Zeit erwachsen wird. Entgegen allen anderslautenden Äußerungen kann der Jüngste Tag eben doch noch nicht angebrochen sein, wenn

54 Ebd.; Martin Luther, Daß der freie Wille nichts sei. Antwort D. Martin Luthers an Erasmus von Rotterdam, übers. von Bruno Jordahn, München ${ }^{3} 1962$ (= Ausgewählte Werke, hg. von Hans H. Borcherdt, München 1934-1983, Ergänzungsreihe, Bd. I, S. 17). Vgl. hierzu Vf., Warum Frauen.

55 Hierzu Walter Dietze, Kuhlmann, S. 239; Wilhelm Schmidt-Biggemann, Apokalypse, S. $214 \mathrm{f}$. 
die Feder noch kratzt, die ihn oder sie be- oder eben erschreiben soll. Und so macht Kuhlmann das einzig konsequente: Er bricht den Kühlpsalter ab. ${ }^{56}$

56 Hierzu Walter Dietze, Kuhlmann, S. 290. 